\title{
Habilidades potenciadas con e-Sport League Of Legends: diseño de caso único
}

\section{Enhanced skills with e-Sport League Of Legends: unique case design}

\author{
Christian Rodríguez González \\ Universidad de Oviedo \\ chris.rg87@gmail.com \\ María Esther Del Moral Pérez \\ Universidad de Oviedo \\ emoral@uniovi.es
}

Recibido: 6/06/2018

Aceptado: 19/06/2018

Publicado: 29/06/2018

\section{RESUMEN}

Los e-Sports son videojuegos multijugador de gran éxito con un marcado carácter competitivo, que priman estrategias cooperativas y tomas de decisiones consensuadas, exigiendo un papel activo a los jugadores para ganar a sus rivales. La presente investigación analiza en qué medida el apoyo de un entrenador experto permite a los jugadores $(\mathrm{N}=10)$ de un e-Sport -como el League of Legends-, desarrollar sus habilidades lógico-matemáticas y entrenarlos para la toma de decisiones adecuadas a través del juego cooperativo. La metodología mixta adoptada permitió: a) contrastar el incremento de las habilidades de los sujetos del grupo experimental (GE) que recibió entrenamiento sobre el videojuego durante 8 sesiones y los del grupo de control (GC) que no, utilizando un test y postest; y, b) analizar las interacciones y decisiones primadas en el juego cooperativo. Los resultados muestran la mejora en las habilidades del GE frente al GC, subrayando el papel clave desempeñado por el entrenador.

\section{PALABRAS CLAVE}

e-Sports; Videojuegos; Aprendizaje cooperativo.

\section{ABSTRACT}

The e-Sports are multiplayer games of great success with a marked competitive character, which prioritize co-operative strategies and consensus decision-making, demanding an active role for the players to win over their rivals. The present investigation analyzes to what extent the support of an expert technician enables the players $(\mathrm{N}=10)$ of an e-Sport -like League of Legends-, develops their logical and mathematical skills for decision making through cooperative play. The mixed methodology adopted allowed: a) contrast the increase of subject's skills of the experimental group (EG) that received training about the videogame during 8 sessions and those of the control group (CG) that did not, using a test and posttest; and b) analyze the interactions and primary decisions in the cooperative game. The results show the improvement in the skills of the EG against the $\mathrm{CG}$, underlining the key role played by the coach.

\section{KEYWORDS}

e-Sports; Videogames; Cooperative learning.

\section{CITA RECOMENDADA}

Rodríguez, C. y Del Moral, M.E. (2018). Habilidades potenciadas con e-Sport League Of Legends: diseño de caso único. RIITE. Revista Interuniversitaria de Investigación en Tecnología Educativa, 4, 28-40. Doi: http://dx.doi.org/10.6018/riite/2018/333771 


\section{Principales aportaciones del artículo y futuras líneas de investigación:}

- Análisis de las habilidades lógico-matemáticas potenciadas y la toma de decisiones mediante los eSports

- Estudio del aprendizaje colaborativo en los e-Sports

\section{INTRODUCCIÓN}

Los videojuegos se han consolidado como una de las industrias orientadas al ocio que mejores cifras de facturación tiene (Benito, 2006; Melchor, 2017), pese a su reciente aparición, los videojuegos online son los que están liderando este sector y transformando esta industria según las demandas de los usuarios. Cada vez más, esta modalidad de videojuegos online está orientándose hacia su vertiente más competitiva con los denominados e-Sports (Martí, 2010).

Los e-Sports son videojuegos centrados en el desarrollo de actividades deportivas donde se produce un entrenamiento físico y mental mediante el empleo de las TIC. Estos juegos han mostrado una evolución impulsada principalmente por la globalización del acceso a Internet, permitiendo que cualquier persona con una conexión a la red pueda disfrutar de ellos, ya sea en la faceta de jugador o de espectador (Asociación empresarial de los e-Sports, 2018). El fácil acceso al juego online ha provocado un incremento considerable del número de consumidores del ocio digital, -especialmente entre los jóvenes-, aunque se está ampliando la ratio de edades (Antón, 2018). Sin embargo, a pesar de ser un negocio con fines eminentemente lúdicos, se están analizado las oportunidades educativas que este tipo de tecnologías virtuales -al servicio del entretenimiento-, brindan implícitamente al desarrollo de diversas habilidades, con objeto de explotar todo su potencial (Pérez-Latorre, 2012).

Así, al margen de su éxito como negocio de reconocido éxito en el contexto mundial, y de considerarse como un fenómeno de consumo de masas virtual, Wagner (2006) -alejándose de su mera condición lúdica- rescata la potencialidad del uso de los e-Sports como herramientas para impulsar diferentes competencias y habilidades en los participantes. Este autor apuesta por un entrenamiento o intervención sistemática, orientada a activar concretamente las habilidades lógico-matemáticas de los sujetos y a mejorar sus tomas de decisiones, puesto que la mayoría de estos juegos exigen la adopción rápida de estrategias eficaces, ya sea de forma individual o cooperativa, para resolver problemas de diversa índole, y con ello dotar de valor añadido a las partidas de juego casual.

En ese sentido, para garantizar la activación de estas habilidades en los jugadores ligada a la toma de decisiones correcta es necesario introducir un instructor -experto jugador-con el fin de optimizar el juego cooperativo y mostrarles las estrategias más idóneas desde un punto de vista externo. De esta forma, el escenario lúdico del videojuego pasa a convertirse en un escenario de entrenamiento de habilidades. En este caso, la presencia del instructor es comparable a la figura del mentor en contextos formativos virtuales, quien organiza y proporciona los recursos didácticos adecuados a los aprendices, mostrándoles sus debilidades y fortalezas, y estableciendo fórmulas alternativas para atender a la diversidad cognitiva (Tejada, Garay y Romero, 2017).

Concretamente, la presente investigación se centra en analizar en qué medida el apoyo permanente de un agente externo, instructor o entrenador permite a los jugadores de un eSport -como el League of Legends-, desarrollar sus habilidades lógico-matemáticas y entrenarlos para la toma de decisiones adecuadas a través del juego cooperativo. La incorporación de este entrenador responde a la necesidad de convertir el escenario de juego en un escenario para adquirir habilidades de forma organizada y planificada. Su misión es tutelar el proceso de aprendizaje derivado del juego cooperativo online mediante una 
intervención sistematizada y un asesoramiento permanente de forma semejante a como se hace en los entornos virtuales de aprendizaje convencionales (Seoane y García-Peñalvo, 2014). Con ello se pretende crear un clima de confianza óptimo para favorecer la interacción entre jugadores que repercuta en la toma de decisiones consensuadas acertadas.

\section{LOS E-SPORTS COMO HERRAMIENTAS EDUCATIVAS: EL CASO DE LEAGUE OF LEGENDS}

Los videojuegos son instrumentos de entretenimiento que gozan de gran popularidad entre el público juvenil gracias a la capacidad de interacción que ofrecen y a su dinamismo (GómezGarcía, Planells y Chicharro-Merayo, 2017). Pero, además pueden suscitar aprendizajes implícitos ligados al desarrollo de habilidades como la resolución de problemas y el fomento del razonamiento deductivo (Revuelta y Guerra 2012), siempre que vaya acompañado de un entrenamiento. Igualmente, Del Moral y Guzmán (2014) señalan que hay videojuegos online como el CityVille- que priman las habilidades lógico-matemáticas, es decir, aquellas relacionadas con la resolución de problemas de organización lógica y matemática, así como la capacidad científica y las destrezas viso-espaciales (Antunes, 2011), lo que les convierte en escenarios óptimos para el aprendizaje. Del Moral, Guzmán y Fernández (2018) comprobaron que algunos serious games utilizados sistemáticamente producían una mejora cualitativa y cuantitativa generalizada de la inteligencia lógico-matemática de los sujetos. Por su parte, Gros (2014) señala que determinados videojuegos presentan situaciones que requieren un estudio exhaustivo para poder continuar con el juego, fomentando que los jugadores examinen sus ideas e hipótesis a partir del análisis de la información recopilada, con objeto de resolver los problemas planteados activando el razonamiento lógico, con lo que se convierten en laboratorios para el entrenamiento de estrategias.

Por otro lado, McGonigal (2011) considera que los juegos multijugador alientan a trabajar en equipo y a aprender juntos, favoreciendo el desarrollo de habilidades sociales y motivando a los jugadores a adoptar un papel activo. En la mayoría de los e-Sports se prima el juego cooperativo donde los miembros de cada equipo deben tomar decisiones consensuadas, de ahí que puedan servir de plataforma para fomentar el aprendizaje cooperativo, a partir de la toma de decisiones fruto de la reflexión previa, el análisis comparativo y la previsión de las consecuencias que de ello se derivan, así como para entender los roles que deben adoptar los jugadores para ser eficaces. Alarcón y Collazos (2017) señalan que videojuegos como League of Legends impulsan el juego grupal, la creatividad y la agilidad mental, donde únicamente es posible ganar si existe una cooperación real entre los jugadores.

En general, los videojuegos se constituyen en escenarios lúdicos que enseñan a tomar decisiones sin las presiones, responsabilidades y consecuencias que éstas podrían acarrear, recreando situaciones semejantes a las de la vida real (Gros, 2014). Evidentemente, el proceso de toma de decisiones implica una compleja actividad cognitiva que supone tanto la evaluación de distintas alternativas para ejecutar una tarea, como la justificación y razonamiento de la opción seleccionada (Artieta y González, 1998). Algunos videojuegos pueden ayudar a simular tomas de decisiones y a visibilizar sus repercusiones, con lo que el jugador aprende a reconducir su juego en nuevas partidas, adoptando las decisiones más acertadas para reducir los impactos negativos de los errores cometidos anteriormente.

Tomar decisiones exige al sujeto activar habilidades complementarias como analizar, clasificar, estimar probabilidades, observar alternativas y decidir, etc., todas ellas susceptibles de entrenamiento. En los e-Sports este proceso permite mejorar la velocidad con la que se estiman las probabilidades de éxito y las alternativas -en función de la decisión tomada- y sobre todo, mejorar las decisiones adoptadas. En este sentido, Woods, Griffiths, Chappell y Davies (2004) señala que el proceso de toma de decisiones y resolución de los problemas planteados -en las misiones de algunos videojuegos- suscita gran interés, ya que puede ayudar a explicar 
a los jugadores cuáles son las mejores opciones, cómo realizarlas, cómo se deben ejecutar las tareas para resolver con éxito los conflictos planteados, etc.

Así pues, el papel de un entrenador o instructor resulta clave en los e-Sports para que los videojugadores adquieran las técnicas y destrezas requeridas para ganar. Éste permite ayudarles a identificar sus debilidades para paliarlas, y a destacar sus fortalezas para potenciarlas, al tiempo que les proporciona la motivación necesaria para progresar. Según Mutanya (2016), la incorporación de un entrenador en la infraestructura de los equipos de eSports resulta ser un componente crítico para el éxito in-game. La complejidad de las misiones u objetivos y el carácter competitivo de los e-Sports, precisa que los videojugadores estén asistidos por un instructor que les proporcione pautas eficaces para adoptar las estrategias más adecuadas para ganar.

En este sentido, League of Legends es un juego cooperativo complejo, donde la toma de decisiones entre los diferentes miembros de los equipos debe ser consensuada y responder a un proceso de aprendizaje colectivo, y al entrenamiento de determinadas habilidades. Por ello, para garantizar una mayor eficacia en las misiones propuestas por el juego se precisa contar con la figura de un instructor que propicie ese proceso generando nuevos conocimientos para los jugadores.

\section{DESCRIPCIÓN DEL VIDEOJUEGO LEAGUE OF LEGENDS}

El e-Sport seleccionado para el presente estudio es el League of Legends (LoL), un videojuego multijugador online del género MOBA (Multiplayer Online Battle Arena) de la compañía americana RIOT Games, de gran popularidad, que posee más de 100 millones de usuarios registrados, una media diaria de 27 millones de jugadores y un pico diario de 7,5 millones de usuarios jugando simultáneamente, cuyas partidas profesionales son seguidas por miles de personas a través de plataformas como TwitchTV, cuenta con comentaristas propios (casters) en varios idiomas y con millones de seguidores por todo el mundo, siendo el evento de los Worlds 2017 récord de audiencia, con más de 106 millones de espectadores (Naranjo, 2017). El juego es gratuito y está disponible en varios idiomas, entre ellos el español.

League of Legends es un juego de estrategia por equipos (5 versus 5), en donde se prima el trabajo cooperativo con el fin de destruir el nexo enemigo ubicado en el centro de su base, y evitar que los otros destruyan el propio. Al inicio de la partida cada jugador escoge un personaje-avatar (entre 120) con unas habilidades y fortalezas propias, y se conforman los equipos, los cuales se ubican en una base en un área concreta del mapa. Dentro de su base se encuentra el nexo, que es el objeto a proteger para no ser derribado, pues -de otro modo- el equipo pierde y se concluye la partida. Desde el nexo aparecen oleadas de súbditos enemigos cada 30 segundos, que se dirigen a través de las líneas (superior, central e inferior) del mapa hacia la base contraria.

El mapa tiene tres carriles, uno central (Mid), otro superior (Top) y otro inferior, por donde se pueden desplazar los jugadores, también existe un territorio neutral llamado jungla. Los jugadores se sitúan: uno en la línea del top, otro en la jungla colindante a su parte del mapa, otro en la línea de mid y los otros dos en la línea de bot: EI ADC (Attack Damage Carry) es el jugador encargado de hacer más daños a los rivales y el Support es el encargado de proteger al resto del equipo (Figura 1). 
Figura 1. Grieta del invocador (mapa del League of Legends)

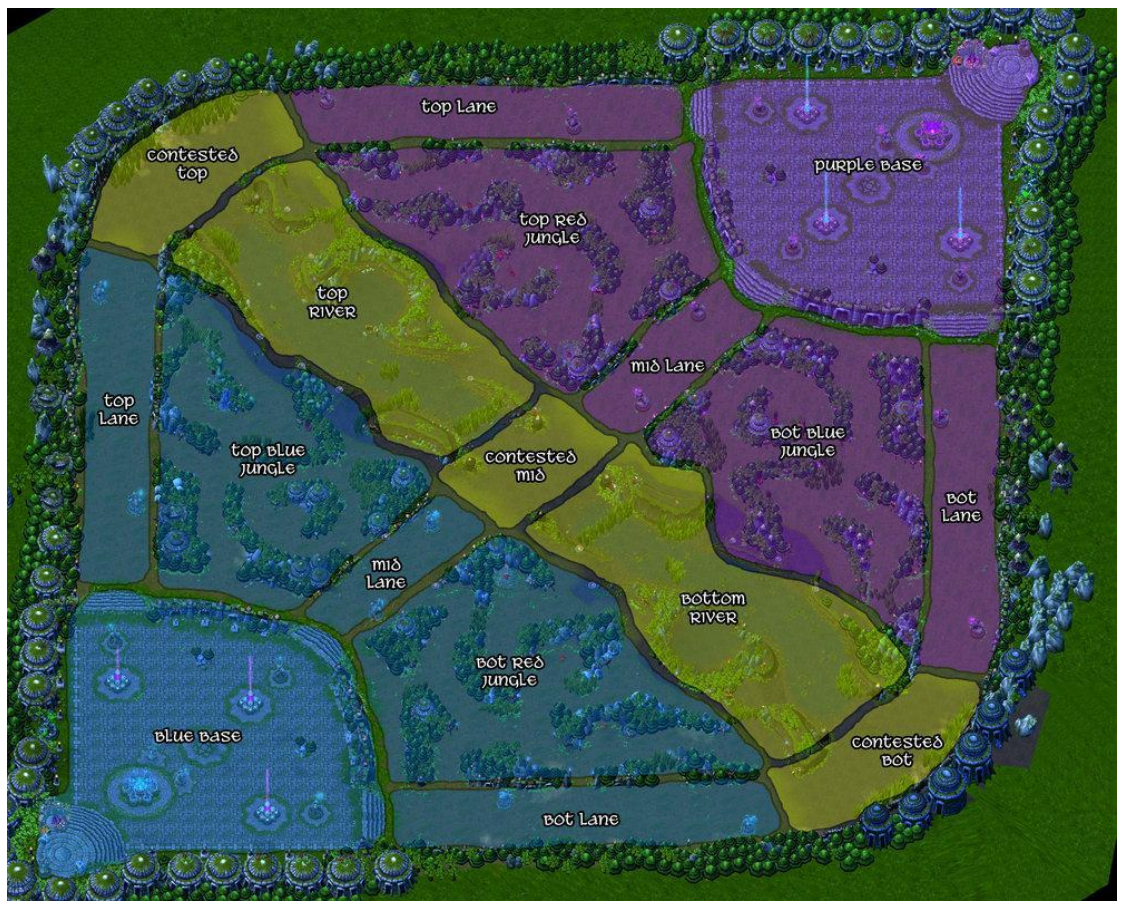

Mecánicas y dinámicas del juego:

Las partidas comienzan en igualdad de condiciones, todos los jugadores tienen la misma cantidad de oro y experiencia inicial, por lo que su habilidad y las decisiones que tomen a lo largo de la partida es lo que determinará la victoria. Cada cual consigue oro automáticamente tras realizar determinadas tareas (eliminar súbditos enemigos o neutrales, derribar torretas enemigas, aniquilar a otros jugadores), y lo puede invertir en la compra de objetos para mejorar las estadísticas de los campeones y ser más fuertes que los rivales. Simultáneamente, aparecen nuevos objetivos (misiones) neutrales en el mapa que pueden proporcionar oro, mejoras permanentes o temporales para el jugador (o equipo) que los consigue. Lograr más oro implica tener mejor economía que el rival, lo que se suele traducir en victoria segura, al incrementar las estadísticas de los personajes se pueden conseguir más objetivos y alcanzar la base rival para destruir el nexo enemigo.

El éxito del juego se apoya en la comunicación y cooperación mutua entre los jugadores, para seleccionar las estrategias de juego más adecuadas y tomar las decisiones más acertadas para acabar con el enemigo y ganar la partida. Indudablemente, la figura de un entrenador garantiza un aprendizaje de las mecánicas y dinámicas del juego más ágil y eficaz, lo que contribuye a mejorar cualitativamente las habilidades de los jugadores como se ha demostrado con la presente investigación.

\section{MÉTODO}

La investigación adopta una metodología mixta:

a) Cuantitativa, focalizada en el uso de test y post-test, para determinar el efecto de la participación en prácticas lúdicas guiadas -por un instructor con el League of Legends, en el incremento de habilidades lógico-matemáticas en los jugadores, estableciendo a ese respecto un grupo de control y otro experimental. En concreto, se puso el foco de análisis en estas habilidades al relacionarse tanto con el factor matemático del juego, ligado a la economía in-game de los equipos y jugadores, como con las 
demandas que se hace a cada jugador para contrarrestar situaciones críticas, exigiéndole gestionar las misiones u objetivos en la partida.

b) Cualitativa, centrada en la observación de los comportamientos y formas de comunicación e interacción de los participantes en el transcurso de las partidas, para analizar -mediante una parrilla para el registro- cómo se produce la toma de decisiones a lo largo del juego y cómo tiene lugar el aprendizaje a partir del juego cooperativo.

\subsection{Proceso}

En un primer momento, se procedió a la asignación aleatoria de los sujetos al grupo de control y al experimental, en base a la generación de una serie de números aleatorios, donde 5 individuos conforman el grupo de control $(\mathrm{N} / 2<5)$ y otros 5 el grupo experimental $(\mathrm{N} / 2 \geq 5)$. $\mathrm{Y}$ se atendió a las variables: edad, sexo, nivel de estudios, experiencia en videojuegos, experiencia en videojuegos online $\mathrm{u}$ otros e-Sports, experiencia en otros MOBA y experiencia previa en el juego de League of Legends. El resto de participantes constituyeron el grupo de control, cabe mencionar que, pese a que algún participante de ambos grupos tenía experiencia previa con el League of Legends, ninguno había gozado de la asistencia de un entrenador que analizase las partidas ni le hiciese ver los fallos cometidos, factor determinante a la hora de dominar las claves de este juego.

\subsection{Objetivos}

Se analiza en qué medida un e-Sports, en concreto League of Legends, puede convertirse en un instrumento para favorecer el aprendizaje cooperativo y la toma de decisiones de los sujetos, así como para activar las habilidades lógico-matemáticas implicadas en las ejecuciones del juego, a partir de la asistencia sistemática de un entrenador que asesora a los jugadores sobre la pertinencia de las decisiones adoptadas. Los objetivos se centran en:

a) Verificar que el juego ayuda a mejorar las habilidades lógico-matemáticas de los jugadores tras participar en la experiencia lúdica guiada por un instructor.

b) Comprobar cómo League of Legends contribuye a favorecer el aprendizaje cooperativo a través del juego.

c) Constatar en qué medida League of Legends influye en la toma de decisiones adecuadas para tener éxito en el transcurso de la partida.

\subsection{Instrumentos}

Se elaboró un cuestionario personal que sirvió para identificar las siguientes variables: sexo, edad, nivel de estudios, experiencia en videojuegos (bastante, media y poca), experiencia con videojuegos competitivos online (e-Sports), experiencia con otros MOBA (Multiplayer Online Battle Arena), experiencia previa con el LoL (League of Legends). Asimismo, para acometer los objetivos de la investigación se utilizó:

a) El Test Mensa (Mensa España, 2016) y el cuestionario de Razonamiento Lógico Matemático (Zeballos, 2015) para evaluar las habilidades lógico-matemáticas antes y después de la intervención, ya que miden la capacidad de los sujetos relativas a las dimensiones lógicas, numéricas y verbales, incluyendo problemas de razonamiento que requieren la organización de elementos presentados gráficamente para poder extraer las soluciones. La prueba consta de 10 ítems que inciden en el razonamiento lógico-matemático, medidos de forma numérica con puntuaciones de 0 hasta el 10.

b) Parrilla para el registro de observación, atendiendo a distintos aspectos:

El aprendizaje cooperativo suscitado con el juego: 
- Comunicación vía chat: ํㅡ de intervenciones por participante, nํ de interacciones entre individuos (pair to pair), formas de cooperación (solicitud de ayuda directa o si acudía voluntariamente a ayudar al otro), manejo del vocabulario del juego.

Las tomas de decisiones efectuadas durante el juego:

- Verbalización conjunta de la situación inicial de la partida: necesidad de tomar decisiones, identificar la actuación a seguir, decisión consensuada.

- Identificación del líder coordinador de la partida y formas de motivar al resto de miembros del equipo.

- Asunción de las propuestas del líder para acometer las estrategias adecuadas para alcanzar la meta propuesta.

- Traspaso del rol de líder a otro miembro más valioso para situaciones concretas.

- Consenso para seleccionar personajes en función de sus fortalezas para crear un equipo con una táctica elaborada.

\subsection{Muestra}

Se seleccionaron 10 individuos entre 18 y 25 años y se el instructor -experto en el juegoles impartió unas sesiones específicas para dominar el juego, ligadas al desarrollo de habilidades. La muestra estuvo integrada por un $90 \%$ de hombres y un $10 \%$ mujeres, quienes aceptaron voluntariamente a participar en la investigación por afinidad e interés por el juego. El $60 \%$ tenía 23 años, el 20\% tenía 25 años, un 10\% contaba con 24 años y otro 10\% tenía 18 años. El $60 \%$ contaba con estudios universitarios o FP de grado superior, un $30 \%$ tenía estudios de Bachillerato o FP de grado medio y solo un $10 \%$ había realizado la ESO. A su juicio, el $70 \%$ manifestó tener bastante experiencia con los videojuegos, un $20 \%$ jugaba de forma casual (media) y un $10 \%$ tenía poca experiencia. Sólo tenían conocimiento de e-Sports o MOBA's unos pocos (4 participantes).

\subsection{Proceso: fases de la investigación y experimentación}

Fase I: Se preparó un cuestionario que serviría para conocer su perfil (edad, sexo, nivel de estudios y conocimientos de videojuegos), y se elaboró un acuerdo de confidencialidad que firmaron los participantes, para asegurar su anonimato y que la investigación fuese rigurosa. Se seleccionó el test que debían contestar los participantes (antes y después). Además, un experto en el juego diseñó un tutorial al respecto, quien sería el encargado de asesorarles durante toda la experiencia lúdica guiada. Se les solicitó que confirmaran online su participación y, se agendaron por consenso las sesiones de juego (las tardes del último fin de semana de abril y las de los fines de semana de mayo). Se adoptó como medio de comunicación Skype, y se les pidió que descargarán el League of Legends. Se les explicó que el resultado de las partidas no era relevante, si no las decisiones tomadas y sus modos de interacción.

Fase II: En la fecha indicada se contactó con los participantes para comentarles cómo se iba a realizar la investigación y asignarles al grupo de control o al grupo de tratamiento. Tras visionar el tutorial y firmar el acuerdo de confidencialidad, se reunieron físicamente con el equipo investigador para realizar el pre-test y recibir instrucciones precisas sobre el juego. Los participantes subsanaron sus dudas tras ver el tutorial del juego que, pese a no ser muy completo, explica las nociones básicas del mismo. Y, el grupo experimental realizaría la primera sesión práctica. El grupo de control no intervino hasta la realización del post-test.

Fase III: Las sesiones prácticas constaron de 2 ó 3 partidas, entre 25 y 50 minutos cada una. Inicialmente, los investigadores y el grupo experimental se reunieron vía Skype para facilitar la comunicación, se pudo registrar sistemáticamente las conversaciones y los comportamientos que resultaban relevantes. Tras iniciar la partida, se observaba y escuchaba a los jugadores, analizando sus formas de interacción. Al final de la partida, y con el 
asesoramiento del entrenador se comentaron las decisiones acertadas, el motivo por el que lo eran, los errores y sus causas, y se les mostraban otras opciones válidas. Concluida la última sesión, el grupo de control y el experimental se reunieron de nuevo con el equipo investigador para realizar el post-test y dar por concluida así su participación. Finalmente, se les agradeció su compromiso y disponibilidad.

Fase IV: Recogida y análisis de los datos: la observación sistemática sirvió para constatar cómo se comunicaban y cooperaban los jugadores durante la partida. Asimismo, los datos obtenidos derivados del cuestionario inicial y de los tests (antes y después) se trataron estadísticamente con el programa STATA, con el fin de contrastarlos y analizarlos para proceder a formular los resultados más significativos de la experiencia.

En concreto, el estadístico utilizado para las variables no categóricas (edad, años de educación y nota del pre-test), fue la $t$ de Student de diferencias de medias de población para muestras independientes. Para las variables categóricas (experiencia en juegos, experiencia en MOBA y experiencia en el LoL), se utilizó un test de suma de rango de Wilcoxon, dado que la muestra era muy pequeña y el test de Wilcoxon es un test no paramétrico que no asume ninguna forma de la distribución. Para las variables de sexo y experiencia online, se utilizó un test de diferencia de proporciones.

\section{RESULTADOS}

\subsection{Habilidades lógico-matemáticas desarrolladas}

La tabla 1 muestra que las características del grupo de control y del grupo experimental son similares en el pretest, antes de iniciar la experiencia lúdica guiada por el instructor. Observándose que no se encuentran diferencias estadísticamente significativas entre las medias de ambos grupos para un nivel de confianza del 95\%, lo que implica que ambos grupos parten de situaciones semejantes en relación con las variables que se analizan y no hay sesgos que pudieran condicionar los resultados a priori.

Tabla 1. Diferencias observadas entre el grupo de control y el grupo experimental.

\begin{tabular}{|c|c|c|c|c|}
\hline $\begin{array}{l}\text { Variables de } \\
\text { observación }\end{array}$ & $\begin{array}{l}\text { Grupo experimental } \\
\qquad(\mathrm{N}=5)\end{array}$ & $\begin{array}{l}\text { Grupo de control } \\
\qquad(\mathrm{N}=5)\end{array}$ & Diferencia & $\begin{array}{l}\text { Estadístico de } \\
\text { análisis }\end{array}$ \\
\hline Sexo & 0.8 & 1 & -0.2 & -1.0541 \\
\hline Edad & 22.4 & 23.6 & -1.2 & -0.9733 \\
\hline Educación & 19.6 & 22 & -2.4 & -1.3720 \\
\hline \multicolumn{5}{|l|}{ Experiencia juegos } \\
\hline Bastante & 0.4 & 1 & -0.6 & 1.936 \\
\hline Media & 0.4 & 0 & 0.4 & \\
\hline Poca & 0.2 & 0 & 0.2 & \\
\hline Ninguna & 0 & 0 & 0 & \\
\hline Experiencia online & 1 & 1 & 0 & - \\
\hline \multicolumn{5}{|l|}{ Experiencia "MOBA" } \\
\hline Bastante & 0.4 & 0.8 & -0.4 & 1.225 \\
\hline Media & 0 & 0 & 0 & \\
\hline
\end{tabular}


$\begin{array}{llll}\text { Poca } & 0.6 & 0.2 & 0.4\end{array}$

$\begin{array}{llll}\text { Ninguna } & 0 & 0 & 0\end{array}$

Experiencia "LoL"

$\begin{array}{rccc}\text { Bastante } & 0.4 & 0.4 & 0 \\ \text { Media } & 0 & 0 & 0 \\ \text { Poca } & 0.2 & 0.6 & -0.4 \\ \text { Ninguna } & 0.4 & 0 & 0.4\end{array}$

Nota pre test

7.8

7.4

0.4

0.6325

Notas: * Diferencias significativas al 5\%. La columna Estadístico de análisis, presenta el valor estadístico de cada test, este es el valor que se compara frente al valor crítico de la distribución t de Student.

La tabla 2 presenta las diferencias existentes entre los sujetos de ambos grupos respecto a la variable habilidad lógico-matemática tras someterlos al post-test, concluida la intervención de la experiencia lúdica guiada por el entrenador.

Tabla 2. Impacto de la intervención guiada con el videojuego en las habilidades lógico-matemáticas

\begin{tabular}{|c|c|c|c|c|}
\hline $\begin{array}{l}\text { Variables de } \\
\text { resultado }\end{array}$ & $\begin{array}{l}\text { Grupo experimental } \\
\qquad(\mathrm{N}=5)\end{array}$ & $\begin{array}{l}\text { Grupo de control } \\
\qquad(\mathrm{N}=5)\end{array}$ & Diferencia & $\begin{array}{l}\text { Estadístico de } \\
\text { análisis }\end{array}$ \\
\hline Nota pre test & 7.8 & 7.4 & 0.4 & 0.6325 \\
\hline Nota post test & 9.2 & 7.2 & 2 & $2.8868^{*}$ \\
\hline
\end{tabular}

Notas: * Diferencias significativas al $5 \%$.

La diferencia entre la nota media del post-test en el grupo experimental frente a la del de control asciende a 2 puntos, siendo estadísticamente significativa al $5 \%$. Por lo tanto, y gracias a la asignación aleatoria del tratamiento, se puede concluir que la intervención con este videojuego online ha mejorado la habilidad lógico-matemática de los participantes del grupo experimental. Hay que destacar que si la intervención del entrenador, -que guiaba las jugadas, comentaba los aciertos y fallos y les daba consejos sobre cómo debían haber actuado-, se hubiera realizado con todos los jugadores, no podría asegurarse que el cambio cualitativo en los resultados finales se debiera a la intervención del mismo. Aun así, bien pudieran existir otros factores que interfirieron en los resultados del post-test, lo que impide certificar categóricamente la causalidad de la intervención.

Para realizar una estimación más ajustada -o contrafactual- del impacto de la intervención se debería haber observado los resultados de cada individuo cuando participa o cuando no lo hace en la instrucción del e-Sport. Pero al no ser posible cuantificar esas dos situaciones en el mismo momento, se optó por comparar los resultados del grupo experimental con los del grupo de control de semejantes características, estableciendo como única diferencia la intervención sistemática del entrenador para dirigir la experiencia lúdica en el experimental. Por lo que, a pesar de todo, se puede afirmar que el cambio cualitativo en los resultados relativos a las habilidades lógico-matemáticas puede estar relacionado con la intervención y participación sistematizada del entrenador. 


\subsection{Fomento del aprendizaje cooperativo y toma de decisiones consensuadas}

A lo largo del experimento, el equipo investigador tuvo acceso a las diferentes partidas, donde pudo comprobar la forma que tenían los jugadores para comunicarse, trabajar en equipo, cómo tomaban las decisiones, quién ejercía el liderazgo y qué participantes se mostraban más activos durante el proceso.

Así, se pudo constatar que los participantes aprendieron a utilizar correctamente el vocabulario específico del juego con las pautas iniciales del entrenador. Casi todos -salvo unotuvieron un número de intervenciones similar durante la partida y se relacionaban con fluidez. A veces pedían de modo explícito asistencia a sus compañeros, y otras, tomaban la iniciativa para ayudar a los otros sin necesidad de que se lo solicitasen. La comunicación pair to pair era la forma más frecuente de interacción entre los individuos que jugaban en la línea inferior, ya que sus roles requieren una comunicación continua. Sin embargo, se observó que uno de los individuos mostraba un número de interacciones mucho menor respecto al resto, por lo que en un intento de minimizar el problema, se dieron pautas para que sus compañeros adoptaran actitudes más asertivas para ponderar sus intervenciones, agradecer la información que compartía sobre la partida, y animarle a interactuar más, lo que se generó interacciones más frecuentes y productivas, que redundaban en el logro de mayores éxitos al favorecer la cooperación mutua.

Tras la verbalización y presentación conjunta de la situación inicial del juego, se daba comienzo a la partida. Los participantes tomaban las primeras decisiones, como seleccionar los personajes más idóneos, dotados de poderes, que supuestamente podían garantizar afrontar los embates enemigos en la partida. Además, tenían claro que debían actuar como un equipo, primando el interés colectivo frente al individual, estaban concienciados de que la toma de decisiones en la partida tenía interés grupal, por ello durante los primeros compases de la partida, todos consensuaban las decisiones, exponiendo lo que a su juicio era más oportuno en determinado momento.

Inicialmente, el líder (rol de support) fue propuesto por el instructor, dotándolo de plena libertad para controlar el mapa y dar información a los demás, y asignándole el papel de coordinador de la partida y de motivador del resto de miembros del equipo. Este rol le otorgaba el privilegio de tomar las decisiones claves, tras escuchar los enfoques de cada jugador. Y los demás debían obedecerle sin cuestionarlas, pues el entrenador les indicó que el éxito en el juego radicaba en la cooperación mutua para alcanzar los objetivos de forma conjunta. Además, podían señalar otro líder, traspasando este rol -en situaciones concretas, como en batallas grupales- a otro miembro más valioso, a quien igualmente debían obedecer, asumiendo sus directrices para culminar la partida con éxito. La clave estaba en proteger al jugador con el rol de ADC, aún a costa de sacrificar a su propio personaje. Así, se pudo constatar cómo todos los miembros del equipo secundaban sus propuestas para alcanzar la meta, -aunque quizá no fuesen las más acertadas-, pero conscientes de que debían operar de forma conjunta. Siempre buscaban el consenso para seleccionar los personajes más idóneos, atendiendo a sus fortalezas, para crear un equipo con una táctica que garantizara el logro de los objetivos.

Los resultados positivos, relativos al ejercicio del juego cooperativo y la activación de las tomas de decisiones, se explican a partir de la intervención del entrenador que les proporcionaba las pautas y consejos para lograr los objetivos del juego. El hecho de jugar al videojuego sin razonar el motivo de la toma de decisiones podría ser insuficiente para haber obtenido estos resultados, lo que puede implicar, que sea necesaria una intervención externa que oriente sobre cómo han de actuar en la toma de decisiones los jugadores, del mismo modo que sucede en otros juegos y/o deportes competitivos en contextos presenciales. 


\section{CONCLUSIONES Y DISCUSIÓN}

A tenor de lo expuesto, se puede decir que la intervención del instructor fue determinante al guiar el juego con el League of Legends, pues contribuyó a mejorar las habilidades lógicomatemáticas, el aprendizaje cooperativo y la toma de decisiones de los jugadores. De forma similar, Carvajal (2014) concluye que este tipo de videojuegos -tutelados por la presencia de un entrenador- favorecen el desarrollo cognitivo del cerebro de los sujetos a partir de las indicaciones efectuadas sobre los fallos cometidos, pues ello les permite mejorar no sólo en la velocidad de reacción para tomar decisiones consensuadas sino en su acierto al jugar de forma cooperativa.

Si bien League of Legends es un e-Sport eminentemente lúdico, sin embargo, puede convertirse en una herramienta para entrenar las habilidades de los jugadores promoviendo la cooperación al interactuar en un espacio virtual común. Además, el papel de un entrenador resulta clave para pautar las intervenciones y orientar a los jugadores sobre las decisiones más idóneas para ganar. El juego cooperativo propuesto por este e-Sport pone a prueba las habilidades lógico-matemáticas de los jugadores a partir de las tomas de decisiones de forma consensuada, además de suscitar numerosos aprendizajes implícitos en un entorno lúdico y competitivo que activa su motivación.

Finalmente, convencidos de la utilidad de los videojuegos (Pérez-Latorre, 2012; Del Moral y Guzmán, 2014, 2015) al propiciar la adquisición de distintas habilidades, creemos que desde el ámbito educativo se deberían aprovechar las oportunidades que ofrecen. Algunos e-Sports fomentan la toma de decisiones y el trabajo cooperativo, otros contribuyen al desarrollo de las inteligencias múltiples (Del Moral, Guzmán y Fernández, 2018), etc. Quizás, dado el alto nivel de motivación que generan, la apuesta por la metodología del Aprendizaje Basado en Juegos (ABJ) (Prensky, 2001; Tobias, Fletcher y Wind, 2014), orientada a integrarlos de forma sistemática y guiada en los contextos formales, podría incrementar en los aprendices las diferentes habilidades a partir de los desafíos o misiones que proponen.

\section{ENLACES}

El pretest y el postest se han extraído de:

- Mensa España (2016). Test lógico-matemáticos. Recuperado de: https://www.mensa.es/

- Zeballos, A. (2015). Test de Razonamiento lógico matemático. Recuperado de: http://razonamiento-logico-problemas.blogspot.com

- Cuestionario personal: https://drive.google.com/open?id=1EPCKrM5OAK7P7L578QitViqWiTiD0MXv

- Registro de observación: https://drive.google.com/open?id=1khTXSnp3W4\|4mrPR2mgUpdlOWx3oA70

\section{REFERENCIAS BIBLIOGRÁFICAS}

Asociación Empresarial de e-Sports (2018). Guía de los e-Sports: Retos de negocio y claves jurídicas. Madrid: AEeS.

Alarcón, I. y Collazos, A. (2017). League of Legends como estrategia para desarrollar la creatividad, el trabajo en equipo, la agilidad mental y el pensamiento estratégico de los estudiantes de la Corporación Universitaria del Huila. En J. Escobar Gómez (Ed.), Las competencias y la gestión del conocimiento (pp. 288-305). Medellín: Editorial Corporación CIMTED. 
Antón, M. (17 de abril de 2018). Los e-Sports ya tienen más público joven que los deportes tradicionales. GeneraciónPro. Recuperado de https://www.cuatro.com/

Antunes, C. (2011). Estimular las Inteligencias Múltiples. Madrid: Narcea.

Artieta, I. y González Labra, M.J. (1998). La toma de decisiones. En M.J. González Labra (Ed.), Introducción a la Psicología del pensamiento (pp. 367-407). Madrid: Trotta Editorial.

Benito, J. (2006). El mercado del videojuego: unas cifras. Revista Icono 14, 4(1), 36-47. doi: https://doi.org/10.7195/ri14.v4i1.396

Carvajal, D. (2014). El papel de los videojuegos en el desarrollo cognitivo. En J. Sierra, y D. Rodrigues (Eds). Contenidos digitales en la era de la sociedad conectada. (pp. 163-178). Madrid: Fragua. doi: https://doi.org/10.13140/2.1.1597.1527

Del Moral, M.E. y Guzmán, A.P. (2014). CityVille: collaborative game play, communication and skill development in social networks. NAER: Journal of New Approaches in Educational Research, 3(1), 31-39. doi: https://doi.org/10.7821/naer.2018.1.248

Del Moral, M.E. y Guzmán, A.P. (2015). CityVille: Promoviendo un juego colaborativo prosocial no bélico en redes sociales. Historia y Comunicación Social, 20(2), 305-326. Recuperado de http://revistas.ucm.es/index.php/HICS

Del Moral, M.E., Guzmán, A.P. y Fernández, L.C. (2018). Game-Based Learning: Increasing the Logical-Mathematical, Naturalistic, and Linguistic Learning Levels of Primary School Students. NAER: Journal of New Approaches in Educational Research, 7(1), 31-39. https://doi.org/10.7821/naer.2018.1.248

Gómez-García, S., Planells, A. J., y Chicharro-Merayo, M. (2017). ¿Los alumnos quieren aprender con videojuegos? Lo que opinan sus usuarios del potencial educativo de este medio. Educar, 53(1), 49-66. https://doi.org/10.5565/rev/educar.848

Gómez-Martín, M., Gómez-Martín, P. y González-Calero, P. (2004). Aprendizaje basado en juegos. Revista Icono 14, 2(4), 1-13. doi: https://doi.org/10.7195/ri14.v2i2.436

Gros, B. (2014). Digital Games in Education, Journal of Research on Technology in Education, 40(1), 23-38. doi: https://doi.org/10.1080/15391523.2007.10782494

Martí, J. (2010). Marketing y videojuegos: product placement, in-game advertising y advergaming. Madrid: ESIC

McGonigal, J. (2011). Reality is Broken: Why Games Make Us Better and How They Can Change the World. New York: The Penguin Press.

Melchor, A. (2017). E-Sports: la nueva era de la competición deportiva. (Tesina de grado). Universidad de Sevilla, Sevilla.

Mensa España (2016). Test lógico-matemáticos. Recuperado de https://www.mensa.es/

Mutanya, A. (14 de enero de 2016). El valor de un coach (v 2.0). 3DJuegos e-Sports. Recuperado de http://www.trasgo.net/

Naranjo, J. (6 de noviembre de 2017). 75 millones de personas vieron la final de los Worlds 2017. E-Sports Marca. Recuperado de https://esports.marca.com/

Pérez-Latorre, Ó. (2012). Del Ajedrez a Starcraft. Análisis comparativo de juegos tradicionales y videojuegos. Comunicar, 38, 121-129. doi: https://doi.org/10.3916/C38-2012-03-03

Prensky, M. (2001). Digital game-based learning. New York: McGraw-Hill.

Revuelta, F.I. y Guerra, J. (2012). ¿Qué aprendo con videojuegos? Una perspectiva de metaaprendizaje del videojugador. RED. Revista de Educación a Distancia, 33. Recuperado de http://www.um.es/ead/red/33

Seoane, A.M. y García-Peñalvo, F.J. (2014). Patrones pedagógicos y docencia en red. En J. A. Jerónimo Montes (Ed.), Formación en red: aprender con tecnologías digitales (pp. 30-47). México: Universidad Nacional Autónoma de México. 
Tejada, E., Garay, U., y Romero, A. (2017). Influencia del mentoring en el rendimiento de los participantes en las comunidades virtuales de práctica. Revista Interuniversitaria de Investigación en Tecnología Educativa, 3, 40-50.

Tobias S., Fletcher J.D., y Wind A.P. (2014) Game-Based Learning. In J. Spector, M. Merrill, J. Elen y M. Bishop (Eds.), Handbook of Research on Educational Communications and Technology. (pp- 485-503). New York, Springer. 485-503.

Wagner, M. (2006, June). On the Scientific Relevance of e-Sports. In J. Arreymbi et al. (eds.). Proceeding of The International Conference on Internet Computing. June 26-29, 2006. Las Vegas, Nevada, USA: Danube University Krems.

Woods, R., Griffiths, M., Chappell, D., y Davies, M. (2004). The structural characteristics of video games: A psycho-structural analysis. CyberPsychology \& behavior, 7(1), 1-10. doi: https://doi.org/10.1089/109493104322820057

Zeballos, A. (2015). Test de Razonamiento lógico matemático. Recuperado de http://razonamiento-logico-problemas.blogspot.com

\section{INFORMACIÓN SOBRE LOS AUTORES}

\section{Christian Rodríguez González \\ Universidad de Oviedo}

Graduado en Magisterio de Educación Primaria con mención en inglés por la Facultad Padre Ossó (Universidad de Oviedo) y Máster en Educación y TIC (eLearning): Investigación por la Universitat Oberta de Catalunya, con experiencia docente en la etapa de Educación Primaria. Líneas de investigación: eSports y nuevas tecnologías en educación, métodos y técnicas de investigación en educación.

\section{María Esther Del Moral Pérez \\ Universidad de Oviedo}

Catedrática de Didáctica y Organización Escolar de la Universidad de Oviedo (España). Imparte Tecnologías de la Información y Comunicación (TIC) aplicadas a la Educación. Facultad de Formación del Profesorado y Educación. Docente de los Másteres de Investigación e Innovación en Educación Infantil y Primaria, e Intervención e Investigación Socio-educativa.

\section{(1) (1) (-)}

Los textos publicados en esta revista están sujetos a una licencia de Reconocimiento 4.0 España de Creative Commons. Puede copiarlos, distribuirlos, comunicarlos públicamente y hacer obras derivadas siempre que reconozca los créditos de las obras (autoría, nombre de la revista, institución editora) de la manera especificada por los autores o por la revista. La licencia completa se puede consultar en:Licencia Creative Commons Atribución-NoComercial-Compartir por igual 4.0 Internacional. 